\title{
Framework and Challenges: H.265/HEVC Rate Control in Real- time Transmission over 5G Mobile Networks
}

\author{
Lei Chen \\ Department of Information \\ Technology \\ Georgia Southern University \\ Statesboro GA 30458, USA \\ lchen@georgiasouthern.edu
}

\author{
Ming Yang \\ Department of Information \\ Technology \\ Kennesaw State University \\ Marietta, GA, USA \\ ming.yang@kennesaw.edu
}

\author{
Luguo Hao \\ College of Info. Eng. \\ Guangdong University of \\ Technology \\ Guangzhou, China \\ haoluguo@hotmail.com
}

\author{
Danda Rawat \\ Eletrical Engineering \& \\ Computer Science Dept. \\ Howard University \\ Washington, D.C., USA \\ db.rawat@ieee.org
}

\begin{abstract}
The newly emerging video coding standard H.265/HEVC, jointly developed by ITU and ISO, is being adopted by an increasing number of applications and platforms such as YouTube, Netflix, video conferencing, live broadcasting, etc. One of the major application scenarios is live broadcasting and real-time streaming over wireless networks, where network adaptability is a major challenge due to the instability/fluctuation of network bandwidth. Rate control has always been a main research area in live video streaming over wireless networks. While rate control has been extensively studied for previous video coding standards, such as H.263 and H.264/AVC, insufficient research has been done for H.265/HEVC. Also, due to the new features, tools, data structures, and algorithms introduced in H.265/HEVC to improve its coding efficiency, the algorithmic and computational complexities of its rate control schemes are greater than all its predecessors. The increasing complexities have hindered the adoption of H.265/HEVC in its real-time streaming over mobile wireless networks, such as $4 \mathrm{G}$ networks and the forthcoming $5 \mathrm{G}$ networks. In this research, the rate control algorithms/methodologies for H.265/HEVC and the corresponding mathematical modeling of each of its components will be investigated. Our goal is to develop low-complexity and highly-efficient rate control schemes for H.265/HEVC to improve its network adaptability and enable its applications to various mobile wireless streaming scenarios. The proposed rate control framework of H.265/HEVC encoder has three major components: precise target rate estimation, accurate bitrate allocation based on textural/motion complexities, and improved rate distortion optimization (RDO). In this paper, we also discuss a number of key research problems and challenges under this framework.

Permission to make digital or hard copies of all or part of this work for personal or classroom use is granted without fee provided that copies are not made or distributed for profit or commercial advantage and that copies bear this notice and the full citation on the first page. To copy otherwise, to republish, to post on servers or to redistribute to lists, requires prior specific permission and/or a fee.

MOBIMEDIA 2017, July 13-14, Chongqing, People's Republic of China Copyright @ 2017 EAI 978-1-63190-156-0
\end{abstract}

Real-time video streaming applications and services are an indispensable part of everyone's work and life nowadays. The technologies supporting such applications are widely adapted in almost all fields; for example, teachers and professors utilize realtime video streaming to deliver lecture contents, and media content providers, such as Netflix and Amazon, stream movies and episodes to their subscribers' devices connected to broadband Internet.

While LANs and WLANs provide higher bandwidth with better reliability, cellular networks give users excellent mobility allowing video streaming in most geographical areas covered by base stations. A study showed that T-Mobile provides average $3 \mathrm{G}$ download speed at $3.84 \mathrm{mpbs}$, compared to its competitors AT\&T at $2.62 \mathrm{Mbps}$, Verizon at $1.05 \mathrm{Mbps}$, and Sprint at $0.59 \mathrm{Mbps}$ across all major U.S. cities [1]. Such bitrates may support the streaming of low quality videos at resolutions up to $480 p(640 \times 480)$. In the world of $4 \mathrm{G}, \mathrm{AT} \& \mathrm{~T}$ leads the competition with an average download rate at $9.12 \mathrm{Mbps}$ [1], theoretically fast enough to support typical 1080p (1920x1080) H.264 HD video streaming [2].

One of the main trends of modern mobile devices is to be equipped with a screen capable of playing videos with 1080p and higher resolutions (e.g. iPad Pro has a display screen with 2732x2048 resolution [3]) such as the $4 \mathrm{~K}(2160 \mathrm{p}, 3840 \times 2160)$. The growing demand for video streaming with higher resolutions poses a challenge to the current $4 \mathrm{G}$ mobile cellular network, given that the best average $4 \mathrm{G}$ user download rate of $9.12 \mathrm{Mbps}$ is much lower than the typical $4 \mathrm{~K}$ video bitrate of $35-45 \mathrm{Mbps}$ at standard frame rate $(24,25,30 \mathrm{fps})[4]$.

Following the Fourth Generation (4G) Terrestrial Mobile Telecommunication standard, approved by the International Telecommunication Union Radio Standards Sector (ITU-R) in January 2012, a new mobile telecommunication generation, the Fifth Generation (5G), entered the stage in the past few years. Over the last four decades, a new Mobile Telecommunication Standard was approved almost every ten years. Based on this schedule, $5 \mathrm{G}$ is expected to be standardized at around 2020. There are the "big three" 5G technologies [5]: ultra-densification, mmWave, and massive multiple-input multiple-output (MIMO). The data rate goals for the $5 \mathrm{G}$ edge rate range from $100 \mathrm{Mbps}$ (easily enough to 
support high-definition streaming) to as much as $1 \mathrm{Gbps}$, and the peak rate is a marketing number, devoid of much meaning to engineers and likely to be in the range of tens of Gbps. On April 13, 2013, H.265/HEVC was approved as an ITU-T standard [6]. In comparison to H.264, H.265/HEVC offers about double the data compression ratio at the same level of video quality. The data rate of $1080 \mathrm{p}$ video using H.264 standard is $4.5 \mathrm{Mbps}$ based on YouTube's report. It is therefore expected that the high-definition video streaming, including 4K UHD (2160p) and $8 \mathrm{~K}$ UHD (4320p), encoded using H.265/HEVC can be transmitted in realtime over the Fifth Generation (5G) cellular networks in the future. Furthermore, until $4 \mathrm{G}$, the succeeding generation systems were basically incremental/extension of their previous generation with certain advancement in terms of data rate and/or latency. However, $5 \mathrm{G}$ is not expected just as an extension of $4 \mathrm{G}$ as incremental approach will not come close to meeting the demands that networks will face by 2020 or so.

Serving as the currently most advanced video coding standard, H.265/HEVC has a major performance advantage over H.264 by reducing about half of the bitrate at all resolutions [7]. Such advantage is a result of several technological advancements over H.264, including finer-grained levels of encoding, flexibility of pixel block-size at various levels, and more accurate intra- and inter-frame predictions. Theoretically, it is expected that standard 4K resolution video streaming encoded with H.265/HEVC will require a bandwidth of around $20 \mathrm{Mbps}$ which should be effortlessly supported by $5 \mathrm{G}$ networks.

Rate fluctuation is a common issue for almost all wireless transmissions, and $5 \mathrm{G}$ networks are not exception. For this reason, it is imperative to wisely control the target bitrate so that it smartly adapts to dynamic network condition and smoothens the receiving and playback at destination. To achieve this goal, at least three important research problems must be further studied: precise target bitrate estimation, accurate bitrate budget allocation, and rate distortion optimization (RDO). Although these issues already existed in previous coding standards and 4G networks, they become almost new challenges in H.265/HEVC and $5 \mathrm{G}$ given the new coding scheme. In the following sections, these three issues will be discussed in greater details.

The rest of this paper is organized as follows: in Section 2, the various rate control approaches for H.265/HEVC and previous standards are discussed; based on this study, a research framework illustrating the logical relationships among the main components is proposed in Section 3; four key research problems are also discussed in this section; in Section 4, we address four major research challenges in the research of H.265/HEVC encoding rate control; conclusion is drawn in Section 5 and references are listed at the end of this paper.

\section{Current Rate Control Approaches}

\subsection{Rate Control Algorithms for Previous Video Encoding Standards}

As early as 1994, L. Wang et al. [8] investigated the rate control problem by using trials and comparisons, e.g. selecting the parameters values through encoding various quantization parameters for best rate control performance. However, this optimization process requires tremendous number of trials before an optimized set of parameter values can be determined, and therefore would not be suitable for real-time video encoding and transmission.

The RM8 rate control algorithm in H.261 was the first video buffer based quantization parameter selection algorithm, which is considered a simple yet practical real-time rate control approach [9]. Following the same path, TM5 algorithm in MPEG-2 attempts to control bitrate by considering both video buffer size and target bitrate. Since then, research on rate control mainly focused on the optimization of encoding parameters through rate distortion models. The two main approaches to Rate Distortion Optimization (RDO) are Dynamic Programming and Lagrange Multiplier.

The RDO-based rate control necessitates finding the optimized rate distortion function, therefore researchers have carefully investigated rate distortion function models, revised and improved the classic model for practical encoder rate control [10]. Based on this method, W. Ding proposed a general rate-quantization function model [11], which became one of the most important and classic rate control models. As shown in (1), $\alpha, \beta, \gamma$ are parameters in this rate control model, and the values may continuously update in the encoding process based on the complexity of video.

$$
\mathrm{R}=\alpha+\frac{\beta}{\mathrm{Q}^{\gamma}} \quad 0<\gamma \leq 2
$$

T. Chiang proposed a second order R-Q model [12], as shown in (2), utilizing the mean quantization parameters of a frame to represent the level of rate distortion. This model has fairly wide range of encoding rate and therefore can be suitable for both encoders based on DCT (discrete cosine transform) and wavelet transform.

$$
R=a Q^{-1}+b Q^{-2}
$$

In 1999, assuming Laplace distribution over video signals, Tihao proposed the second order R-D model for MPEG-4, which became the VM8 rate control algorithm [13]. Based on this algorithm, researchers proposed extensible rate control algorithm that can be used for no-delay-restriction and low-delay-restriction with bufferrestriction constant bitrate (CBR) encoding [14]. Such model can be used along with sliding window for choosing appropriate parameters in order to handle issues caused by video scene changes.

Based on the DCT statistical analysis of residual image, Jodi proposed rate distortion model for both high and low date rates [15], which was later adopted in the H.263+ video standard and finally became the rate control method for TMN8 [16][17]. TMN8, along with TM5 of MPEG-2 and VM8 of MPEG-4, became generally recognized classic rate control algorithms. Brief discussions about these three classic algorithms are given below. 
The TMN8 (Test Model Near-term 8) algorithm has two steps: first, the frame-level rate control determines whether to skip or encode the current frame by setting a threshold $M$ in the video buffer; second, the macro-block rate control calculates the quantization step size of each macro-block using second order R-Q model. The TM5 (Test Model 5) algorithm has three steps: first, bit allocation designates a target bitrate and then allocates bitrate based on the target bitrate of the GOP (group of pictures) and the actual bitrate of a previous similar GOP (I, P and B frames); second, the rate control calculates the bit number of current encoding frame, and then compares this calculated number with target bit number, in order to adjust the quantization parameters for optimized bitrate control; the third step is to calculate the self-adaptive quantization step Q: calculate the mean square deviation of the brightness of the current macro-block, if it is greater than average, then increase quantization step Q and compression rate. The VM8 algorithm is a rate control algorithm based on a second order R-D model, as formulated in (3) below. It first initiates the fixed-value parameters such as target bitrate, frame rate, sequence length, initial buffer size, etc. Then rate allocation is implemented based on available bit number and the actual bit number of the most recent encoded frame. Quantization parameter (QP) is calculated using the second order model and encode parameters are updated after encoding current frame. To prevent buffer overflow, skip to next frame when buffer storage is $80 \%+$ full.

$$
R=\frac{X_{1} \cdot S}{Q P}+\frac{X_{2} \cdot S}{Q P^{2}}
$$

As for H.264, there are mainly two classic rate control algorithms: JVT-G012 [18] and JVT-O016 [19]. The JVT-G012 introduces the concept of BU (Basic Unit), which is a group of continuous macro blocks and can be as large as an entire frame or as small as a macro block. The algorithm estimates the MAD (Mean Absolute Difference) value of the current BU by using linear model based on the MAD value of the BU at the same location in the previous frame. When a larger BU is used, higher PSNR (Peak Signal to Noise Ratio) can be achieved, but with higher fluctuation of bitrate. On the other hand with smaller BU, relatively stable bitrate can be achieved but with lower PSNR. The rate control algorithm is applied to three levels: GOP level, frame level and BU level. The JVT-O016 algorithm utilizes the spatial and temporal relationship of macro-block and improves JVT-G012 by reducing the inaccuracy of model parameters introduced by non-relevant historical data points. While at the GOP and frame levels, it is identical to JVT-G012. JVT-O016 has made noticeable improvements in the initial bitrate estimation of macro-blocks, bitrate-quantization (Q-R) model, distortion-quantization (D-Q) and encoding complexity, and achieved the optimization of macroblock quantization size within an entire frame. With similar complexity, JVT-O016 has achieved almost $1 \mathrm{db}$ improvement on frame PSNR over JVT-G012.

\subsection{H.265/HEVC Rate Control}

The existing rate control algorithms for H.265/HEVC can be divided into three categories: R-Q (Rate-Quantization) modelbased rate control, $\rho$-domain ( $\rho$ is the percentage of zero values within quantization parameters) rate control, and $R-\lambda$ ( $\lambda$ is the Lagrange Multiplier based on image complexity) model-based rate control.

\subsubsection{R-Q Model Rate Control}

The R-Q model is one of the most classic mathematical models for rate control. This type of algorithms are based on the assumption that video bitrate $\mathrm{R}$ is determined entirely on quantization parameters (QPs) of the residual encoding, and through adjusting the QPs, the encoder can achieve target bitrate. This assumption, however, implies that all other configurations and parameters of the encoder, including macro-block mode, prediction mode, and motion evaluation mode are all fixed, and QP is the only tunable parameter. Based on this model, Si et al. proposed a new selfadaptive rate control algorithm addressing the new characteristics and structure of H.265/HEVC [20]. This algorithm controls bitrate of H.265/HEVC encoder at the frame level. It is formulized in (4), where $\mathrm{R}$ is the coding rate, a is the model parameter, $\mathrm{X}$ is complexity of the current frame and QP is the quantization parameter. According to (4), the QP can be calculated using formula (5), where $T_{n}$ is the target bitrate of current frame.

$$
\begin{aligned}
& R=a \times X / Q P \\
& Q P_{n}=\alpha \times X / T_{n}
\end{aligned}
$$

This algorithm estimates the distortion of each frame by defining and utilizing a distortion model $\mathrm{D}=\alpha \times\left(\mathrm{SATD} \times Q P^{\gamma}\right)^{\beta}$, and further smoothens video frames following such distortion estimation. In recent years, Q-P based HEVC algorithms include the frame-level rate control algorithm proposed by Tu et al. in 2014 [21] and the rate-complexity based inner-frame rate control algorithm proposed by Tian and Zhou in the same year [22].

For video encoding standards in the past, encoder has limited flexibility and encoding tools are relatively simple and therefore the R-Q model turned out to have satisfactory performance. However, H.265/HEVC supports various encoding modes, levels, units, prediction modes, and encoding tools and therefore has high level of encoding flexibility, resulting QP not being the only factor for determining output rate. Subsequently, the R-Q model may not be suitable for H.265/HEVC standard.

\subsection{2 $\rho$-domain Rate Control}

This type of rate control algorithms attempts to discover the relationship between data rate $\mathrm{R}$ and $\rho$, the percentage of zero values within quantization parameters, and further control data rate based on such relationship. However, this model has an assumption that $\rho$ and QP has a one-to-one matching relationship. Therefore this model is essentially the same as the aforementioned R-Q model, which determines data rate based on QP. Another major issue with the $\rho$-domain model is its minimum support of various- 
sized blocks, which limits its extension to rate control in H.265/HEVC. For this reason, very limited $\rho$-domain rate control research exists after the release of H.265/HEVC standard.

In 2014, Biatek et al. proposed a $\rho$-domain based rate control model, which obtains the linear relationship between data rate and $\rho$ through experiments and results [23]. Specifically, for obtaining quantization parameters $\mathrm{QP}$, this algorithm first estimates the encoding parameters of the current CTU (Coding Tree Unit) based on the previous CTU, then correlates them with the Laplace distribution of transformation coefficients. Although this algorithm has greatly reduced complexity, it would not be considered suitable for H.265/HECV due to reasons previously addressed.

\subsubsection{R- $\lambda$ Model Rate Control}

As the aforementioned models would not be suitable for H.265/HEVC, many recently proposed rate control algorithms are based on the R- $\lambda$ model, where $\lambda$ is the Lagrange Multiplier based on image complexity. In $2014, \mathrm{Li}$ et al. proposed a new rate control algorithm based on the R- $\lambda$ model [24]. This algorithm calculates the target bitrate based on network bandwidth and buffer status, and then allocates bitrates in three different levels: GOP, frame and CTU. Then finally the rate control is implemented according to (6), where QP is the quantization parameter, $\lambda$ is the Lagrange Polynomial parameter, bpp is the average bit number used for each pixel, and $\mathrm{C} 1, \mathrm{C} 2, \alpha$ and $\beta$ are additional parameters for this model, which will dynamically update during the encoding process. Compared to R-Q model algorithms, the R- $\lambda$ algorithms have significantly improved the PSNR and therefore has been adopted by H.265/HEVC reference software.

$$
Q P=C_{1} \times \ln (\lambda)+C_{2} \quad \lambda=\alpha \times b p p^{\beta}
$$

There have been a number of recently proposed $\mathrm{R}-\lambda$ based algorithms since 2014 . Summaries of the major $\mathrm{R}-\lambda$ algorithms are given below.

In 2014, Yang et al. proposed a low-latency $\mathrm{R}-\lambda$ model based algorithm for H.265/HEVC [25]. This algorithm first estimates target bitrate based on the available most accurate $\mathrm{R}-\lambda$ model, i.e. $\mathrm{R}=\alpha \times \lambda^{\beta}$, then allocates bitrate over GOP, frame and CTU levels. Specifically, bitrate allocated at GOP level is determined based on buffer status and the actual bit consumption of encoded frames. At the frame and CTU levels, the strategy is to first calculate a rate based on buffer status and then calculate a second rate based on the equal allocation principle, finally use a weighted formula to determine the final allocated bitrate based on the above two rates.

Li et al. proposed a weighted R- $\lambda$ model based algorithm in 2014 targeting the applications of video conferencing [26]. In this algorithm, eye-tracking experiments were conducted to help evaluate the relative importance of different areas in video frames, such as backgrounds, faces, etc., in order to create a weighted map for video encoding, where weights are assigned based on the importance for each area. Logically more bits will be allocated to areas with greater weights. This algorithm works effectively in improving the facial details in video conferencing applications.

Wang et al. proposed a gradient-based R- $\lambda$ model algorithm in 2015 for inner-frame encoding rate control [27]. In HEVC, inter-frame encoding efficiency has already been greatly improved, and therefore inner-frame encoding rate control is highly demanded. In their algorithm, the Gradient Per Pixel (GPP) concept is used to effectively describe frame complexity and to obtain an accurate Lagrange Multiplier $\lambda$ and initial encoding quantization parameters QP, which further help accurately allocate bitrate and improve the performance of $\mathrm{R}-\lambda$ algorithm.

In 2015, Xie et al. proposed a rate control and allocation model that is based on temporal domain complexity [28]. The algorithm utilizes the classic R- $\lambda$ model for its rate control, while for rate allocation, it not only considers texture complexity but further takes into account the motion complexity in the temporal domain, which overcomes certain limitations in existing algorithms.

Wen et al. proposed a new R- $\lambda$ model based rate control algorithm in 2015 that estimates the characteristics of Largest Coding Unit (LCU) based on the pre-encoding of $16 \times 16 \mathrm{CU}$, in order to obtain more accurate R- $\lambda$ model parameters before the actual encoding starts [29]. Experiments show that this algorithm improves video quality.

Guo et al. proposed an $\mathrm{R}-\lambda$ rate control algorithm based on picture complexity, particularly targeting the encoding of television videos where sudden changes exist in pictures [30]. This algorithm makes use of a frame layer sliding window, within which a quick motion evaluation (ME) is conducted for each frame so as to evaluate the picture complexity using ME. Specifically, when the picture complexity of a frame is greater than three times of the average complexity of previous frames, the current frame is then considered to contain sudden changes and subsequently more bits are allocated for encoding this frame. During the encoding process, $\lambda$ value is dynamically adjusted to achieve precise rate control.

In 2015, Li et al. proposed an $\mathrm{R}-\lambda$ rate control algorithm using Recursive Taylor Expansion (RTE) to achieve optimized bit allocation at LCU level [31]. By studying these proposed R- $\lambda$ model rate control algorithms, we consider that the $\mathrm{R}-\lambda$ model would be more suitable for H.265/HEVC compared to previous models and therefore should be the direction of current and future research in H.265/HEVC rate control.

\subsubsection{Other H.265/HEVC Rate Control Algorithms}

R-Q model, $\rho$-domain model and R- $\lambda$ model are among the three most important rate control models for H.265/HEVC and previous encoding standards. Summarized below are a number of other types of HEVC rate control algorithms.

In 2013, Si et al. proposed a rate control algorithm targeting the CTU level [32]. In H.265/HEVC encoding, CTU is the smallest encoding unit that uses an independent QP in a frame. This algorithm attempts to establish a model using the relationships among quantization parameters, Laplace distribution parameters of 
residual signals after transformation from the original signals, and the Lagrange multiplier. The quantization parameters are then dynamically adjusted based on the Laplace distribution characteristics of transformation residuals for each CTU. Combining with the advantages of frame level rate control algorithms, this algorithm has achieved more precise and accurate rate control and encoding. Experimental results show that this algorithm performs better in encoding H.265/HEVC than the rate control algorithm as part of the standard proposals of JCTVCH0213 and JCTVC-K0103.
Based on the traditional Rate Distortion Optimization (RDO), Li et al. proposed a new algorithm Delay-RDO (dRDO), which considers how the delays at each different part of the route (including video encoding delay, encoding buffer delay, signal transmission and propagation delay, decoding buffer delay and video decoding delay) contribute to the overall end-to-end delay [33]. By analyzing and considering the variance of the delays, data rate provided by sources and the signal redundancy bits, the traditional RDO can further be optimized.

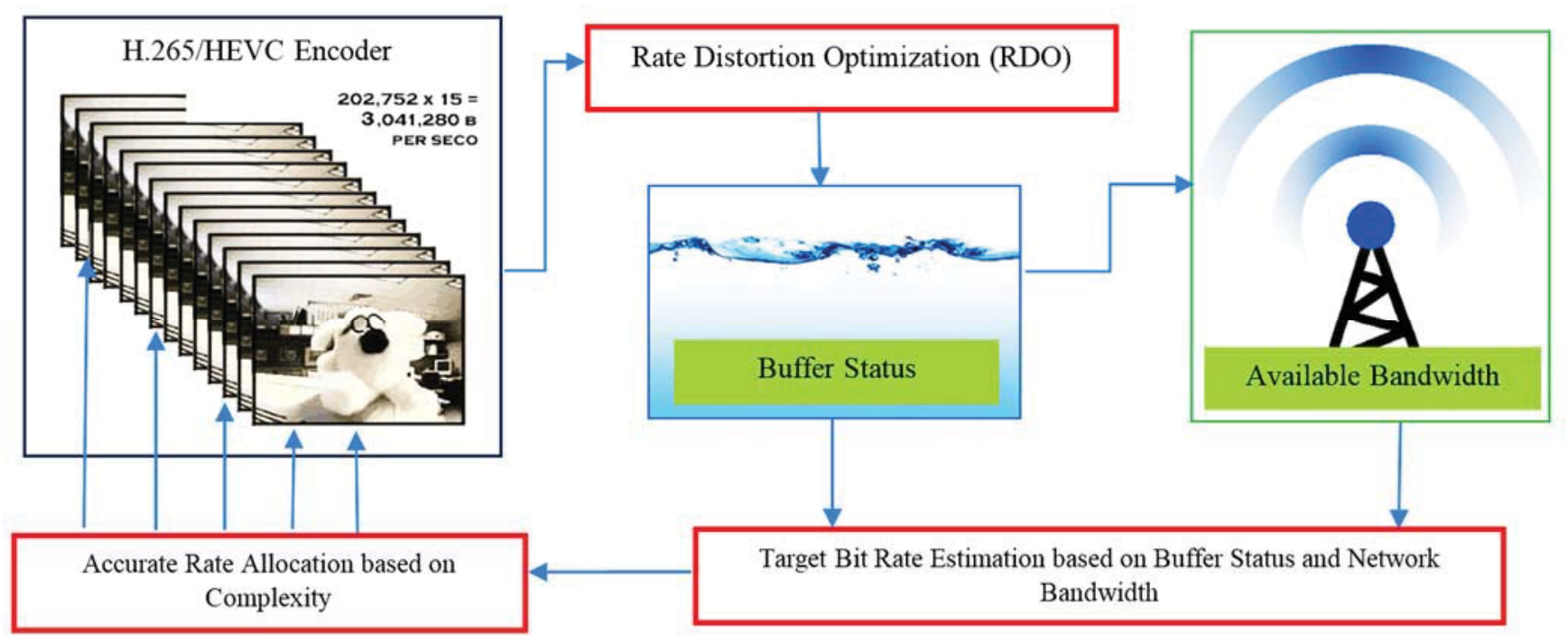

Figure 1: Proposed Framework for H.265/HEVC Real-time Video Encoding and Delivery in Mobile Networks

The texture complexity of video is one of the indicators of image complexity and should be a factor to consider for target bitrate estimation. Sun et al. discovered the quasi-linear relationship between the texture complexity at the Largest Coding Unit (LCU) level and encoding data rate, and in 2014 they proposed a method of estimating video texture complexity based on the relationship between spatial domain and temporal domain for optimizing the rate control of encoding [34]. In (7), Rleft is the estimated number of bits for encoding the $i^{\text {th }}$ and other unencoded Basic Units (BU) in the current frame. $C_{L C U}^{i}$ is the texture complexity of the $\mathrm{i}^{\text {th }} \mathrm{BU}$, and $\mathrm{N}$ is the total number of BUs in the current frame. Experimental results indicate that this proposed algorithm performs well in controlling bitrates and buffer overflow.

$$
R_{L C U}^{i}=R_{\text {left }} \cdot \frac{C_{L C U}^{i}}{\sum_{k=i}^{N} C_{L C U}^{k}}
$$

In 2015, Chao et al. introduced an algorithm that divides macro blocks into groups based on their various characteristics and assigns different quantization parameters to different groups [35]. Experimental results show that this algorithm can both retain the gradient characteristics and fulfill the requirement of obtaining relatively accurate target bitrate.

\section{H.265/HEVC Rate Control Research Framework and Key Research Problems}

In this section, we propose a research framework that visually illustrates the logical relationship among the research problems and components in $5 \mathrm{G}$ mobile networks. Further discussions on the challenges are presented in the following section.

In order to precisely control output data rate, the first step is to accurately estimate the target H.265/HEVC output bitrate for transmission in mobile network. Subsequently, allocate bitrate within the various levels of H.265/HEVC, including GOP, frame, slice and CTU. Additionally, establish an optimized mathematical model for rate control, including residual distortion optimization (RDO), based on the studies of existing models and algorithms. Finally, test the model and algorithm in real 5G mobile network environment and adjust the values of parameters for optimization.

The proposed framework is illustrated in Figure 1, where the H.265/HEVC encoder outputs data to video buffer which connects to the mobile networks for real-time video streaming delivery. 
Buffer can be considered as a pool holding water and the output data stream from encoder can be considered as water flowing into the pool. The water flowing out from the pool in this case would be the buffer output data streaming to the mobile communication network. Based on this model, rate control can be viewed as controlling the incoming and outgoing water flows such that the pool water level is within an acceptable range, e.g. not too high or not too low. Therefore, H.265/HEVC encoding rate control can be essentially determined with the consideration of both the buffer status and the available bandwidth of mobile network.

We consider four key research problems in this model: 1) determining the target bitrate of encoder; 2) logical and precise allocation of target bitrate; 3) rate control algorithm; 4) strategy of rate control particular for mobile networks. These four key research problems are further discussed in the rest of this section.

The target bitrate of the encoder is essentially important as it is the baseline bitrate that rate control algorithm can function upon. Based on the framework proposed, this value should be mostly determined by the buffer size and available network bandwidth. Additionally, the buffer is a dynamic mechanism that coordinates with the encoder output bitrate and the current available bandwidth. More specifically, while the buffer is receiving data from the encoder and sending out data to the mobile network, its status (how full it is) should also be proactively provided as feedback to the target bitrate estimation process and consequently affects forthcoming encoding data rate. As an example, when the buffer is $80 \%$ full (considered as an alert level) and the current available bandwidth is lower than the current encoder output bitrate (water flowing out at a lower speed than water flowing in, as in the pool example), the target bitrate estimation algorithm should then decrease the target bitrate for the forthcoming encoder output so that it would not overflow the buffer. In another scenario where buffer is also $80 \%$ full but the current available bandwidth is significantly greater than encoder output data rate, then the target bitrate either does not need to be changed or can even be increased to provide better video quality. For this key research problem, it is important to find the mathematical model that coordinates all these variables with appropriate parameters.

The logical and precise allocation of target bitrate ties closely to the new encoding structure introduced in H.265/HEVC that is quite different from previous encoding standards. Specifically, the allocation mechanism should consider allocating bits in various levels, including GOP (Group of Pictures), frame, slice (a unit between GOP and CTU), CTU (Coding Tree Unit), and even the lower levels such as CB (Coding Block) and PB/TB (Prediction Block or Transform Block). Currently, most bitrate allocation algorithms reach down to CTU level using even allocation strategy. We consider that picture complexity and algorithm complexity should be both considered in order to achieve dynamic, precise and fine-grained bit allocation.

In our research framework, the rate control algorithm functions in the Rate Distortion Optimization (RDO) module, which consists of a few sub-modules. H.265/HEVC encoding mode RDO includes block mode, estimation mode and quantization mode, through which an overall optimized encoding mode can be determined that can subsequently help determine rate control algorithm. Another consideration is the RDO of optimizing individual parameters in the algorithm which is a very challenging task and therefore most current algorithms focus on only optimizing QP.

The strategy of rate control for mobile networks includes the study of characteristics of the forthcoming $5 \mathrm{G}$ mobile networks as the patterns of the variation and availability of bandwidth can play an important role in rate control of the encoder. Therefore this should be a part of the rate control algorithm.

\section{H.265/HEVC Rate Control Research Challenges}

Based on the aforementioned research framework and the four key research problems, there are four challenges that we consider critical in solving the research problems: 1) How to precisely model and obtain real-time buffer status? 2) At which encoding unit level(s) bitrate allocation should be considered? 3) How to consider the RDO of global parameter? 4) How to address and solve the contradictory issue between the performance of rate control and computational complexity? These four challenges are further discussed as follows.

The classic models for describing video buffer are Leaky Bucket (LB) and Token Bucket (TB). LB has constant outgoing data rate while tokens enter a TB at a constant rate. However, in our research model, both of the incoming and outgoing data rates of the video buffer are dynamic and constantly changing. Therefore none of the two models can directly apply and a new mathematical model is needed.

The lower the encoding level is reached, the more precise the allocation of bitrate can be implemented, however on the other hand the complexity of computation will increase. Most current algorithms put their effort in the GOP, frame and CTU levels. We consider that for optimized bitrate control for $5 \mathrm{G}$ networks, lower level of encoding should also be considered since the computation power of mobile and terminal devices have been increasing and will continuously increase. Encoding units/levels of $\mathrm{CTU} / \mathrm{CB} / \mathrm{PB} / \mathrm{TB}$ are all introduced in H.265/HEVC. How to make use of these new data structure and the corresponding motion and texture complexity of encoding units toward precise rate allocation with satisfying performance is a challenge.

The current approaches mostly optimize the single QP parameter in the RDO process. However, this may not be sufficient for high resolution videos, such as HD and UHD, in the $5 \mathrm{G}$ mobile network environment. A major challenge would be how to formulate a global parameter, incorporating other parameters, such as block mode, estimation mode, quantization mode and motion vector, for a comprehensive RDO process.

The better the rate control, the more likely an algorithm would have greater complexity and consequently is more difficult to be implemented. Nonetheless, this challenge ties closely to the 
development of future $5 \mathrm{G}$ mobile networks and mobile devices. We are confident that with the advancement of new mobile and networking technologies, more complex encoding algorithms can be developed and applied to achieve better video quality and more reliable video content delivery over mobile networks.

\section{CONCLUSION}

In this paper, we presented our study of existing rate control approaches for H.265/HEVC and previous standards. Based on this study, we proposed our research framework for H.265/HEVC Realtime video encoding and delivery in mobile networks. Following this framework, four key research problems for rate control are discussed and four research challenges are addressed. While the research problems are still unsolved and the future research is facing the aforementioned challenges, our current research is valuable in that it visibly analyzed and presented the logical relationships among the components in H.265/HEVC real-time encoding rate control, and sheds light on the research directions in this particular domain which may affect everyone's life and work in the near future.

\section{REFERENCES}

[1] M. Sullivan, "3G and 4G Wireless Speed Showdown: Which Networks Are Fastest?", PCWorld, retrieved online from http://www.pcworld.com/article/ 253808/3g and_4g_wireless_speed_showdown_which_networks_are fastest .html on January 5, 2016.

[2] O. Awobuluyi, J. Nightingale, Q. Wang, and J.M. Alcaraz-Calero, "Video Quality in 5G Networks: Context-Aware QoE Management in the SDN Control Plane," in Computer and Information Technology; Ubiquitous Computing and Communications; Dependable, Autonomic and Secure Computing; Pervasive Intelligence and Computing (CIT/IUCC/DASC/PICOM), 2015 IEEE International Conference on, vol., no., pp.1657-1662, 26-28 Oct. 2015.

[3] S. Al-Majeed, S.K. Askar, and M. Fleury, "H.265 Codec over 4G Networks for Telemedicine System Application," in Computer Modelling and Simulation (UKSim), 2014 UKSim-AMSS 16th International Conference on, vol., no., pp.292-297, 26-28 March 2014.

[4] Recommended upload encoding settings for YouTube, Google Support Document, retrieved online from https://support.google.com/youtube/answer/ 1722171 ?hl=en on January 5, 2016.

[5] J. Andrews, S. Buzzi, W. Choi, S. Hanly, A. Lozano, A. Soong, and J. Zhang, "What Will 5G Be?", IEEE Journal on Selected Areas in Communications, Vol.32, No.6, June 2014.

[6] “AAP Recommendation: H.265". ITU, 2013-04-13.

[7] G. Sullivan, J.R. Ohm, W.J. Han, and T. Wiegand, "Overview of the High Efficiency Video Coding (HEVC) Standard", IEEE Transaction on Circuits and Systems for Video Technology, vol. 22, no. 12, December 2012.

[8] L. Wang, "Bitrate control for hybrid DPCM/DCT video codec". IEEE Trans. CSVT.,Vol.4, pp509-517,1994.

[9] ISO/IECJTCI/SC29/WGIIMPEG93/457. MPEG-2 Video Test Model 5. 1993.4

[10] H.M. Hang and J.J Chen, "Source Model for transform video coder and its application part I: fundamental theory", IEEE Trans. CSVT., Vol7(2), pp287$298,1997$.

[11] W. Ding and B. Liu, "Rate control of MPEG video coding and recording by rate-quantization modeling", IEEE Trans. CSVT., Vol.6, pp12-20, Feb. 1996.

[12] T. Chiang and Y.Q. Zhang, "A new rate control scheme using quadratic ratedistortion modeling”, IEEE Trans. CSVT., Vol.7(1), pp246-250, Feb. 1997.
[13] SO/IEC JTC1/SC29/WG11, MPEG-4 video Verification Model V8.0, Maui Dec 1999.

[14] H.J. Lee, T. Chiang, and Y.Q. Zhang, "Scalable rate control for MPEG-4 video. IEEE Trans", CSVT., Vol.10, pp878-894, Feb. 2003.

[15] R.C. Jordi and D. Lei. Rate control in DCT video coding for low-delay communication”, IEEE Trans. CSVT., Vol.9(1), Feb. 1999.

[16] R.C. Jordi, "A frame-layer bit allocation for H.263+. IEEE Trans", CSVT., Vol10(7), pp1154-1158, 2000.

[17] R.C. Jordi, Shawmin Lei, "Rate control for low-delay video communication", ITU-T SG16, q15a20.doc, Portland, Jane 1997.

[18] Li Z.G, Pan F, and Lim K.P, "Adaptive basic unit layer rate control for JVT[C]", JVT-G012, 7th meeting, Pattaya II, Thailand 2003.

[19] W. Yuan, S. Lin and Y. Zhang, "Optimum bit allocation and rate control for H.264/AVC[C]", In Joint Video Team(JVT) of ISO/IEC MPEG \& ITU-T VCEG 15th Meeting, JVT-O016, Busan, KR, 2005.

[20] Junjun Si, Siwei Ma, Xinfeng Zhang, Wen Gao, “Adaptive Rate Control for High Efficiency Video Coding", 2012 IEEE Conference on Visual Communications and Image Processing (VCIP), 27-30 Nov. 2012, San Diego, CA.

[21] Qin Tu, Xiaoqiang Guo, Aidong Men, Ji Qi1 Jun Xu, "A Frame-Level HEVC Rate Control Algorithm for Video With Complex Scene over Wireless Network", Vehicular Technology Conference (VTC Spring), May 2014.

[22] Ling Tian and Yimin Zhou, "A new rate-complexity-QP algorithm (RCQA) for HEVC intra-picture rate control”, 2014 International Conference on Computing, Networking and Communications, Multimedia Computing and Communications Symposium, p375-380.

[23] Biatek, T., Raulet, M., Travers, J.-F., Deforges, O, "Efficient quantization parameter estimation in HEVC based on $\rho$-domain", 2014 Proceedings of the 22nd European Signal Processing Conference (EUSIPCO), 1-5 Sept. 2014, pp 296-300, Lisbon, Portugal.

[24] Bin Li, Houqiang Li, Li Li, Jinlei Zhang, “ $\lambda$ Domain Rate Control Algorithm for High Efficiency Video Coding”, IEEE Transactions on Image Processing, Vol. 23, Issue 9, pp. 3841-3854, 2014.

[25] Zhongzhu Yang, Li Song, Zhengyi Luo and Xiangwen Wang, "Low Delay Rate Control for HEVC", 2014 IEEE International Symposium on Broadband Multimedia Systems and Broadcasting (BMSB 2014), 25-27 June 2014, Beijing, China.

[26] Shengxi Li, Mai Xu, Xin Deng, and Zulin Wang, "Weight-based R- $\lambda$ rate control for perceptual HEVC coding on conversational videos", Signal Processing: Image Communication, Vol. 38, October 2015, pp. 127-140

[27] Miaohui Wang, King Ngi Ngan, Hongliang Li, "An Efficient Frame-Content Based Intra Frame Rate Control for High Efficiency Video Coding", IEEE Signal Processing Letters, Vol. 22, No. 7, July 2015.

[28] Jianfeng Xie, Li Song, RongXie, Zhengyi Luo, Xiangwen Wang, "Temporal dependent bit allocation scheme for rate control in HEVC", 2015 IEEE Workshop on Signal Processing Systems (SiPS), 14-16 Oct. 2015, Hangzhou, China.

[29] Jiangtao Wen, Meiyuan Fang, Minhao Tang, Kuang Wu, "R- $\lambda$ Model Based Improved Rate Control for HEVC withPre-Encoding", 2015 Data Compression Conference.

[30] Y. Guo, B. Li, S. Sun, J. Xu, "Rate control for screen content coding in HEVC", 2015 IEEE International Symposium on Circuits and Systems (ISCAS 2015),Lisbon, May 2015

[31] S. Li, M. Xu, Z. Wang, "A novel method on optimal bit allocation at LCU level for rate control in HEVC", 2015 IEEE International Conference on Multimedia and Expo (ICME),Turin, June 29 2015-July 32015.

[32] Si J.J., Ma S.W., Wang S.Q. and Gao W, "Laplace Distribution Based Rate Control algorithm for HEVC", Journal of Shanghai University (Natural Science), Vol. 19, No. 3, Jun. 2013.

[33] Chenglin Li, Hongkai Xiong, and Dapeng Wu, "Delay-Rate-Distortion Optimized Rate Control for Wireless Video Communication", IEEE International Conference on Image Processing (ICIP), Paris, September 2014.

[34] Heng Sun, Can Zhang, and Shaoshuai Gao, "LCU-Level Bit Allocation for Rate Control in High Efficiency Video Coding", ChinaSIP 2014.

[35] Jianshu Chao, Robert Huitl, Eckehard Steinbach, and Damien Schroeder, "A Novel Rate Control Framework for SIFT/SURF Feature Preservation in H.264/AVC Video Compression", IEEE Transaction on Circuits and System for Video Technology, vol. 25, no. 6, June 2015 